\title{
Control of spin configuration in half-metallic $\mathrm{La}_{0.7} \mathrm{Sr}_{0.3} \mathrm{MnO}_{3}$ nano-structures
}

\author{
J. Rhensius, ${ }^{1,2,3}$ C. A. F. Vaz, ${ }^{1, a)}$ A. Bisig, ${ }^{1,3,7}$ S. Schweitzer, ${ }^{3}$ J. Heidler, ${ }^{1}$ H. S. Körner, ${ }^{1,3}$ \\ A. Locatelli, ${ }^{4}$ M. A. Niño, ${ }^{4}$ M. Weigand,${ }^{5}$ L. Méchin, ${ }^{6}$ F. Gaucher, ${ }^{6}$ E. Goering, ${ }^{7}$ \\ L. J. Heyderman, ${ }^{2}$ and M. Kläui ${ }^{1,3, b)}$ \\ ${ }^{1}$ SwissFEL, Paul Scherrer Institut, Villigen PSI 5232, Switzerland \& Laboratory for Nanomagnetism and Spin \\ Dynamics, Ecole Polytechnique Fédérale de Lausanne (EPFL), Lausanne 1015, Switzerland \\ ${ }^{2}$ Laboratory for Micro- and Nanotechnology, Paul Scherrer Institut, Villigen PSI 5232, Switzerland \\ ${ }^{3}$ Fachbereich Physik, Universität Konstanz, Universitätsstraße 10, Konstanz D-78457, Germany \\ ${ }^{4}$ Sincrotrone Trieste, Basovizza-Trieste 34149, Italy \\ ${ }^{5}$ Helmholtz-Zentrum Berlin für Materialien und Energie GmbH, Berlin 12489, Germany \\ ${ }^{6}$ GREYC, UMR 6072, CNRS - ENSICAEN - UCBN, 6 Boulevard du Maréchal Juin, Caen Cedex 14050, France \\ ${ }^{7}$ Max-Planck-Institute for Intelligent Systems (formerly Metals Research), Heisenbergstrasse 3, Stuttgart \\ 70569, Germany
}

(Received 11 May 2011; accepted 18 July 2011; published online 11 August 2011)

\begin{abstract}
We investigate the interplay between the governing magnetic energy terms in patterned $\mathrm{La}_{0.7} \mathrm{Sr}_{0.3} \mathrm{MnO}_{3}$ (LSMO) elements by direct high-resolution $\mathrm{x}$-ray magnetic microscopy as a function of temperature and geometrical parameters. We show that the magnetic configurations evolve from multidomain to flux-closure states (favored by the shape anisotropy) with decreasing element size, with a thickness-dependent crossover at the micrometer scale. The flux-closure states are stable against thermal excitations up to near the Curie temperature. Our results demonstrate control of the spin state in LSMO elements by judicious choice of the geometry, which is key for spintronics applications requiring high spin-polarizations and robust magnetic states. (C) 2011 American Institute of Physics. [doi:10.1063/1.3623480]
\end{abstract}

Half-metals are characterized by one semiconducting and one metallic spin band, rendering the conduction electrons fully spin-polarized at the Fermi level. This property makes this class of materials ideally suited for applications such as spin-injectors for spintronics devices and magnetic random access memory elements based on the tunnel magnetoresistance effect. ${ }^{1}$ The optimally doped $\mathrm{La}_{0.7} \mathrm{Sr}_{0.3} \mathrm{MnO}_{3}$ (LSMO) is a half-metal with a measured spin polarization of nearly $100 \%,{ }^{2}$ and a critical temperature $T_{c} \approx 370 \mathrm{~K},{ }^{3}$ making it a good candidate for room temperature device applications. ${ }^{4,5}$ LSMO belongs to a class of compounds known as the "colossal" magnetoresistance (CMR) manganites, which are characterized by a rich electronic behavior as a function of chemical doping, strain, and magnetic and electric fields. This sensitivity to external parameters makes the CMR manganites particularly suited for studying a wide range of physical phenomena, including the role of charge carrier density on the spin configuration in multiferroic heterostructures ${ }^{6,7}$ and on the metal to insulator transition, ${ }^{8}$ and the role of strain on the equilibrium magnetic state. ${ }^{9}$ The study of static and dynamic properties of domains and in particular of domain walls in this class of materials is also of interest for applications in mass storage concepts based on domain walls, ${ }^{10,11}$ while the high spin polarization $P$ together with low saturation magnetization $M_{s}$ at temperatures near $T_{c}$ promise a high spin-torque efficiency for current-induced domain wall motion (which scales as $P\left(M_{s}\right){ }^{12}$ For the realization of experiments and applications based on single domains or do-

\footnotetext{
a) Author to whom correspondence should be addressed. Electronic mail: carlos.vaz@cantab.net.

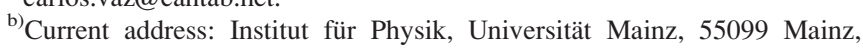
Germany
}

main walls, one needs a suitable system with controllable domain states. This is best accomplished in systems with low magnetocrystalline anisotropies, where the spin configuration can be controlled by shape anisotropy. However, so far, high spin polarization materials such as $\mathrm{Fe}_{3} \mathrm{O}_{4}$ and $\mathrm{CrO}_{2}$ have shown spin structures that are strongly dominated by magnetocrystalline anisotropies, ${ }^{13,14}$ which makes them unsuitable for many devices. In this letter, we study the evolution of the magnetic states of highly spin polarized LSMO thin film elements as a function of size, shape, thickness, and temperature to show that, for sufficiently small elements, the equilibrium states consist of flux closure states, which are stable up to $T_{c}$. Well defined domain walls arise in confined structures, demonstrating that the spin structure can be tailored by the element geometry, making it an ideal candidate for spintronic applications, such as spin-torque based devices.

The LSMO films (15 $\mathrm{nm}$ and $50 \mathrm{~nm}$ in thickness) were deposited by pulsed laser deposition from a stoichiometric target onto $\mathrm{SrTiO}_{3}$ (001) single crystal substrates. The laser radiation energy of the $\mathrm{KrF}$ excimer laser (248 nm) was 220 $\mathrm{mJ}$ at a repetition rate of $3 \mathrm{~Hz}$. The oxygen pressure was 0.35 mbar and the substrate temperature was held at $720^{\circ} \mathrm{C}$ during growth. These parameter values were found to be optimal for producing single-crystalline films with smooth surfaces as judged by x-ray diffraction (XRD) and atomic force microscopy (AFM). The XRD results confirm that the LSMO films are [001]-oriented, while superconducting quantum interference device (SQUID) magnetometry shows that the magnetization is in-plane, with a Curie temperature of about $340 \mathrm{~K}$ (see Fig. 2), typical for good quality films of this composition. The micro- and nano-patterning was carried out with focused ion beam (FIB) lithography, where a focused $\mathrm{Ga}$ ion beam induces local structural changes and 
Ga implantation, driving the LSMO to a non-magnetic state. This effect is used to pattern microscopic structures, by writing non-ferromagnetic lines to define the borders of the element.

$\mathrm{X}$-ray circular magnetic dichroism (XMCD) imaging was performed in order to study the (in-plane) spin configuration of patterned LSMO thin films. Two methods are used, both based on the absorption of circularly polarized photons at the $\mathrm{Mn} \mathrm{L}_{3}$-edge: (1) scanning total electron yield microscopy, where a focused $\mathrm{x}$-ray beam generates photoelectrons that are counted for each scanned position, a newly developed technique ${ }^{15}$ which is well suited for imaging small regions with a high resolution; (2) photoemission electron microscopy (PEEM) ${ }^{16}$ used to image larger areas of the patterned film. The spatial resolution of both techniques is of the order of $30 \mathrm{~nm}$. XMCD images were taken on different element geometries for $15 \mathrm{~nm}$ and $50 \mathrm{~nm}$ thick LSMO films, after magnetizing the samples with a strong initial magnetic field $\mathbf{B}_{\text {Init }}$ of $\sim 1 \mathrm{kG}$.

The evolution of the magnetic state as a function of element size shows a transition from a multidomain state to simpler, shape anisotropy-dominated, magnetic states with decreasing lateral dimension. This is illustrated in Fig. 1 for square and rectangle elements (and in Fig. 3(b) for disc elements). The larger elements have a domain configuration similar to a high moment $\mathrm{C}$-state, but with domains not uniformly magnetized (with ripple contrast) separated by irregular domains walls, which we associate with local pinning of the magnetization that may help stabilize these high magnetic moment configurations. For lateral dimensions below about $2 \mu \mathrm{m}$ and $4 \mu \mathrm{m}$ for the $15 \mathrm{~nm}$ and $50 \mathrm{~nm}$ thick LSMO films, respectively, the magnetic states fall into well-defined shape anisotropy-dominated flux closure states, with uniformly magnetized domains and sharp domain walls, similar to those found in $\mathrm{NiFe}$ and $\mathrm{Co}$ non-oxide materials. ${ }^{17}$ This shows that, at sufficiently small sizes, the magnetostatic energy dominates the micromagnetic configuration of the system and, in particular, that the strength of the pinning sites is smaller than the magnetostatic energy. The fact that the epitaxial LSMO thin films are magnetically soft down to the submicrometer scale is an unexpected result given the presence of epitaxial strain, which tends to introduce strong pinning, and the tendency of these complex oxides to phase segregate. ${ }^{18}$ This magnetically soft behavior is a key prereq-

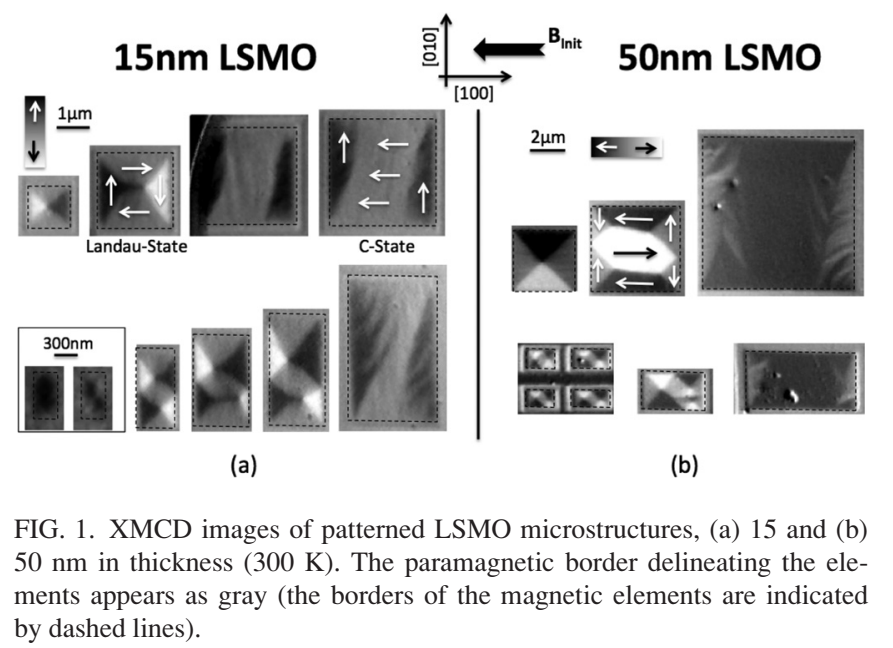

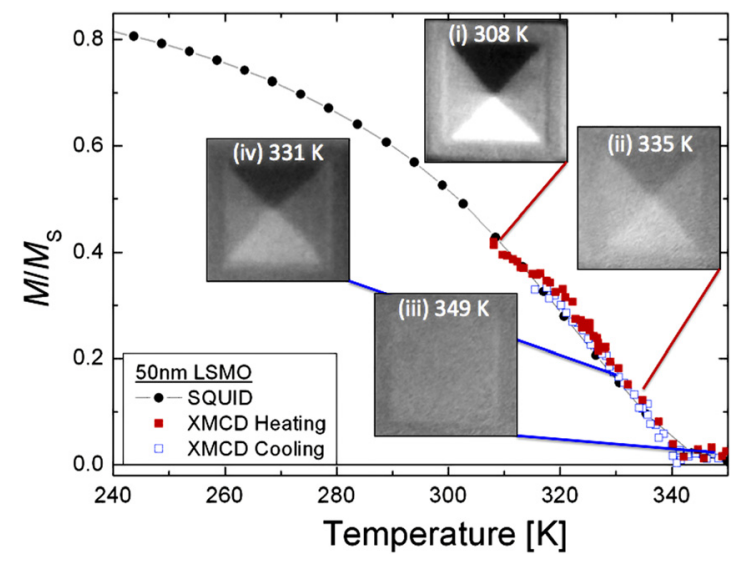

FIG. 2. (Color online) Scaled XMCD contrast vs temperature for a $50 \mathrm{~nm}$ LSMO square element, $3.3 \mu \mathrm{m}$ size (full squares for heating and empty squares for cooling) and SQUID magnetometry measurements (circles), used to calibrate the temperature scale for the XMCD data. The inset shows XMCD images of the domain states at (1) $308 \mathrm{~K}$, (2) $335 \mathrm{~K}$, (3) $349 \mathrm{~K}$, and (4) $331 \mathrm{~K}$ in the cooling cycle.

uisite for use in devices and opens the way for the implementation of high spin-polarized materials. While in epitaxial 3d metals, the magnetocrystalline anisotropy and pinning often dominate, here we find a soft magnetic behavior reminiscent of the softer polycrystalline $3 \mathrm{~d}$ metal structures, but in an epitaxial high spin polarization material. For the smallest elements $(300 \mathrm{~nm} \times 500 \mathrm{~nm}$ rectangles), a transition to quasi-uniform states is observed for the $15 \mathrm{~nm}$ LSMO elements indicating that, at this thickness, the exchange energy cost associated with the presence of domain walls becomes larger than the magnetostatic energy.

For the $15 \mathrm{~nm}$ thick LSMO elements, the Landau states shown in Fig. 1(a) are found to be slightly distorted, with [100]-oriented domains larger than [010] domains. This is interpreted as arising from the presence of a small uniaxial anisotropy contribution, which sets a preferred orientation of the magnetization (confirmed by SQUID magnetometry). The influence of the uniaxial anisotropy can also be seen in the $50 \mathrm{~nm}$ thick sample, where the anisotropy slightly favors [100] domains in the flux closure pattern. AFM data of LSMO films grown on $\mathrm{SrTiO}_{3}$ show the existence of steps, which are expected to induce a magnetic uniaxial anisotropy. ${ }^{19-21}$ The step density in our films is low compared to
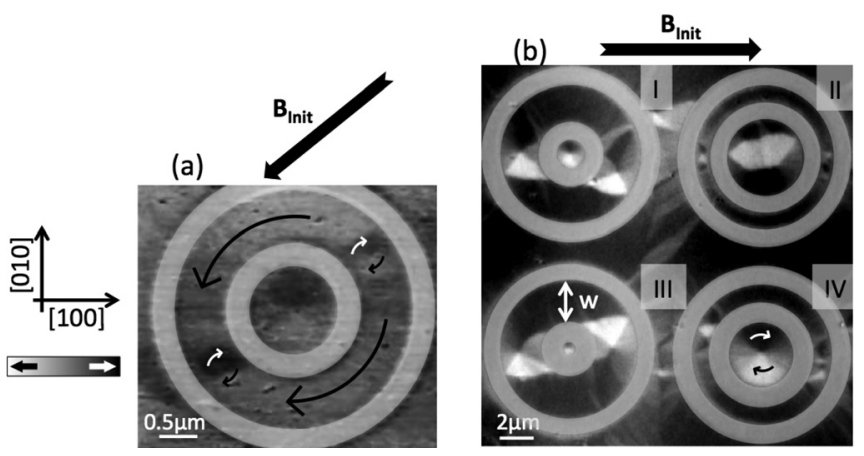

FIG. 3. XMCD images of LSMO rings and discs after saturation (300 K). (a) XMCD-TEY image of a $15 \mathrm{~nm}$ LSMO/STO ring, $620 \mathrm{~nm}$ in width, and of a $1.2 \mu \mathrm{m}$ diameter disc in the center. An onion state is present in the ring, with vortex walls separating the two domains. The central disc is in the vortex state. (b) XMCD image of $50 \mathrm{~nm}$ LSMO ring and disc elements. (To guide the eye, the nonmagnetic area is grayed out.) 
the films investigated in Refs. 19-21, resulting in a small anisotropy contribution and explaining the dominant role of the shape anisotropy in our elements. The tuning of the magnetic anisotropy by appropriately engineering the substrate is then an additional degree of freedom that can be used to control the magnetic state. ${ }^{20-22}$

The thermal stability of the magnetic states was studied by imaging the magnetic states as function of temperature to above $T_{c}$. The result of one such measurement for a Landau state in a $50 \mathrm{~nm}$ thick LSMO square element is shown in Fig. 2. In order to confirm that the lack of XMCD contrast at high temperature corresponds to a non-magnetic state, we compare the XMCD contrast with the results of SQUID magnetization measurements, confirming that for temperatures above $340 \mathrm{~K}$ the system is in a paramagnetic state. This rules out limitations in spatial or temporal resolution as the reason behind the lack of magnetic contrast in PEEM. The images in Fig. 2 show that the Landau state persists up to $\sim 340 \mathrm{~K}$, and is recovered upon cooling from above $T_{c}$, demonstrating that the low energy states are thermally stable up temperatures close to $T_{c}$. Such temperature stability is necessary for spin torque experiments that entail Joule heating. Multidomain states, on the other hand, tend to change with increasing temperature and are not reproduced upon cooling.

Ring elements were used as prototypical structures for the study of domain walls ${ }^{23}$ in confined LSMO structures. In Fig. 3, we present magnetic images of ring elements with widths in the range from $0.2-2.2 \mu \mathrm{m}$. One finds that the rings are in the so-called "onion" state, corresponding to the presence of two domains in a ring, separated by two domain walls. A scanning total electron yield XMCD image of a 15 $\mathrm{nm}$ thick LSMO ring, $650 \mathrm{~nm}$ in width, is shown in Fig. 3(a). For this particular ring dimension, vortex domain walls (short black and white arrows) separate the two domains of the onion state (long black arrows). Fig. 3(b) shows ring elements with widths ranging from $600 \mathrm{~nm}$ (II) up to $2.2 \mu \mathrm{m}$ (III) for the $50 \mathrm{~nm}$ thick LSMO film; the rings favor the formation of vortex walls, although double vortex walls are also observed, as in Fig. 3(b) (IV) (domain wall to the right). These results demonstrate that by tuning the size and geometry of the element, it is possible to control the type of domain wall present in a confined geometry. The magnetic configurations found in our structures can be reproduced well by micromagnetic simulations, ${ }^{24}$ showing that the spin structure in LSMO can be controlled by a suitable choice of the element geometry and that the relevant spin structures, such as well-defined domain walls, can be selectively positioned and controlled in this material, which is a key step to using this material in a device. These results indicate that LSMO follows similar micromagnetic energetics as $3 \mathrm{~d}$ ferromagnetic elements ${ }^{17,23}$ and have the advantage of not only having well-controlled spin structures but also the high spin polarization of a half-metal.

In summary, we have determined the dimensional crossover from multidomain to shape-defined magnetic states in highly spin-polarized LSMO structures. Weak pinning and low magnetic anisotropies give rise to highly symmetric states that are determined by the shape anisotropy. In particular, we show that the well defined domain walls are generated in spatially confined geometries such as rings, whose character can be controlled by varying the width of the element. In addition, we demonstrate that the low energy states are robust against thermal excitation up to the critical temperature. These results show that LSMO is a promising candidate for both the study of fundamental domain wall phenomena in highly spin polarized materials and for device applications, where robust and well determined spin configurations are key.

We would like to thank M. Hagner for support with sample preparation. This work was funded by EU's 7th Framework Programme IFOX (NMP3-LA-2010 246102), MAGWIRE (FP7-ICT-2009-5 257707), the European Research Council through the Starting Independent Researcher Grant MASPIC (ERC-2007-StG 208162), the Swiss National Science Foundation, and the DFG.

${ }^{1} \mathrm{H}$. Hidaka, in Embedded Magnetic RAM, edited by K. Zhang (Springer US, 2009), p. 241.

${ }^{2}$ J.-H. Park, E. Vescovo, H.-J. Kim, C. Kwon, R. Ramesh, and T. Venkatesan, Nature 392, 794 (1998).

${ }^{3}$ G. H. Jonker and J. H. van Santen, Physica 16, 337 (1950).

${ }^{4}$ C. A. F. Vaz, C. H. Ahn, and V. E. Henrich, in Spin-Polarisation Effects in Oxide Materials, edited by A. Hirohata and Y. Otani (Research Signpost, Kerala, 2009), p. 145.

${ }^{5}$ J. Cibert, J.-F. Bobo, and U. Lüders, C. R. Phys. 6, 977 (2005).

${ }^{6}$ H. J. A. Molegraaf, J. Hoffman, C. A. F. Vaz, S. Gariglio, D. van der Marel, C. H. Ahn, and J.-M. Triscone, Adv. Mater. 21, 3470 (2009).

${ }^{7}$ C. A. F. Vaz, J. Hoffman, Y. Segal, J. W. Reiner, R. D. Grober, Z. Zhang, C. H. Ahn, and F. J. Walker, Phys. Rev. Lett. 104, 127202 (2010).

${ }^{8}$ X. Hong, A. Posadas, A. Lin, and C. H. Ahn, Phys. Rev. B 68, 134415 (2003).

${ }^{9}$ Y. Wu, Y. Matsushita, and Y. Suzuki, Phys. Rev. B 64, 220404(R) (2001).

${ }^{10}$ R. Cowburn, U.S. patent WO/2007/132174 (22 November 2007).

${ }^{11}$ S. S. P. Parkin, M. Hayashi, and L. Thomas, Science 320, 190 (2008).

${ }^{12}$ A. Thiaville, Y. Nakatani, J. Miltat, and Y. Suzuki, Europhys. Lett. 69, 990 (2005).

${ }^{13}$ A. Biehler, M. Kläui, M. Fonin, C. König, G. Güntherodt, and U. Rüdiger, Phys. Rev. B 75, 184427 (2007).

${ }^{14}$ M. Fonin, C. Hartung, U. Rüdiger, D. Backes, L. Heyderman, F. Nolting, A. F. Rodríguez, and M. Kläui, J. Appl. Phys. 109, 07D315 -(2011).

${ }^{15}$ D. Nolle, M. Weigand, G. Schütz, and E. Goering, Microsc. Microanal. (in press).

${ }^{16}$ J. Stöhr, Y. Wu, B. D. Hermsmeier, M. G. Samant, G. R. Harp, S. Koranda, D. Dunham, and B. P. Tonner, Science 258, 658 (1993).

${ }^{17}$ M. Kläui and C. A. F. Vaz, in Handbook of Magnetism and Advanced Magnetic Materials, Vol. 2, edited by H. Kronmüller and S. Parkin (Wiley, New York, 2007), p. 879.

${ }^{18}$ E. Dagotto, Science 309, 257 (2005).

${ }^{19}$ M. Mathews, F. M. Postma, J. C. Lodder, R. Jansen, G. Rijnders, and D. H. A. Blank, Appl. Phys. Lett. 87, 242507 (2005).

${ }^{20}$ M. Kubota, T. Taniuchi, R. Yasuhara, H. Kumigashira, M. Oshima, K. Ono, H. Okazaki, T. Wakita, T. Yokoya, H. Akinaga, M. Lippmaa, M. Kawasaki, and H. Koinuma, Appl. Phys. Lett. 91, 182503 (2007).

${ }^{21}$ Y. Takamura, R. V. Chopdekar, A. Scholl, A. Doran, J. A. Liddle, B. Harteneck, and Y. Suzuki, Nano Lett. 6, 1287 (2006).

${ }^{22}$ P. Perna, L. Méchin, M. Saïb, J. Camarero, and S. Flament, New J. Phys. 12, 103033 (2010).

${ }^{23}$ M. Kläui, J. Phys.: Condens. Matter 20, 313001 (2008).

${ }^{24}$ Code available at http://math.nist.gov/oommf/. 